\title{
Carbonation Effect on the Chloride Profile
}

\section{Carmen Andrade}

International Center for Numerical Methods in Engineering (CIMNE), Universitat Politècnica de Catalunya - BarcelonaTECH, Campus Nord UPC, 08034-Barcelona, Spain, candrade@cimne.upc.edu

\begin{abstract}
In natural marine exposures, the chloride profile may have a maximum in the concrete surface (surface concentration) or this maximum may appear some mm or even centimeters, in depth. This fact has been attributed to a) a "washing effect" due the continuous action of the sea water, $b$ ) the wet and dry "convection" induced by the action of the sea water waves c) the leaching of the hydroxide ions or d) the carbonation of the external layer of concrete. In a test in the laboratory, the profile resulting in a diffusion chloride test in most of the cases presents the maximum in the surface of the specimen but seldom has this maximum beyond the surface. This behaviour in natural and in laboratory conditions, although noticed, has not been satisfactorily explained. The author has addressed the problem in a previous communication. In present one shows evidences on the role of the carbonates/bicarbonates in the water of the testing solution. It can be deduced from the analysis of the profiles obtained with de-carbonated solutions that the carbonates/bicarbonates ions compete with the chlorides in the binding with the hydrated cement phases, which results in a modification of the chloride profile and a different diffusion coefficient. The maximum seems to be produced by the higher mobility of the chloride with respect to the carbonate/bicarbonate. The bound chlorides are displaced by the carbonation and the free chlorides move inside forward. In the external parts less total chlorides are noticed because the free chlorides are function of the bound chlorides.
\end{abstract}

Keywords: Concrete, Chlorides, Profile, Maximum, Carbonate.

\section{Introduction}

In marine environments, chlorides penetrate trough the pore network of concrete. This ingress is usually modelled, through the so named "error function equation", assuming pure diffusion, a constant external chloride concentration and a constant diffusion coefficient, in spite that wet-dry cycles are produced in the atmospheric and tidal zones. The chloride profiles obtained can have a decreasing shape from the concrete surface or can present a maximum some $\mathrm{mm}$ inside the concrete (Andrade et al., 1997; Andrade et al., 2015). This "anomalous" profile departing from the fickian behaviour has been attributed to four main causes (Baroghel et al., 2012; Qiang et al., 2011; Lianfang et al., 1999; Ngala et al., 1997; Galan et al., 2015): a) convection due to wet and dry cycling b) leaching of calcium and hydroxides, c) carbonation of the concrete skin or d) that the concrete "skin" has a different microstructure than of the concrete bulk. All these processes may overlap with the pure diffusion. The reasons and the interaction of several of them remain without experimental confirmation. The consequence of this lack of description of the process is one of main limitations for the accuracy of the prediction of concrete service life because the error can be of decades.

Present work contributes to study the effect of carbonation in the production of the profile maximum and in particular, analyses the impact in the chloride testing by pure diffusion (ponding test), because it is studied the effect of de-carbonating the external salt solution by boiling the water. The results show that the carbon dioxide dissolved in the solution 
influences the profile shape giving different diffusion coefficient that could explain part of the scatter in the testing. The results also show that the previous carbonation does not prevent completely the chloride binding, that also impacts in the value of the diffusion coefficient deduced from fitting of the error function equation.

\section{Experimental}

The cement used was an OPC of low $\mathrm{C}_{3 \mathrm{~A}}$ content (CEM I $52.5 \mathrm{~N}-\mathrm{SR}$ ). Its chemical composition is shown in Table 1.

Table 1. Chemical composition (\%) of CEM I 52.5 N-SR 5.

\begin{tabular}{cccccccc}
\hline $\mathrm{SiO}_{2}$ & $\mathrm{Al}_{2} \mathrm{O}_{3}$ & $\mathrm{CaO}$ & $\mathrm{Fe}_{2} \mathrm{O}_{3}$ & $\mathrm{~K}_{2} \mathrm{O}$ & $\mathrm{MgO}$ & $\mathrm{Na}_{2} \mathrm{O}$ & $\mathrm{SrO}$ \\
\hline 19.868 & 3.751 & 64.356 & 4.052 & 0.65 & 0.624 & 0.65 & 0.051 \\
\hline
\end{tabular}

The specimens were of paste in order to avoid the influence of the aggregate proportion in the further analysis. The w/c ratio was of 0.4. Three cylindrical paste specimens of $200 \mathrm{~mm}$ height and $150 \mathrm{~mm}$ diameter were moulded. After setting and hardening for $24 \mathrm{~h}$, the specimens were cured in the humid chamber with humidity higher than $95 \%$ and temperature about $23^{\circ} \mathrm{C}$ for 6 further days. After removing the specimens from the curing chamber, the three specimens were cut horizontally by its half, in order to have the tests by duplicate exposing the same surface (the cut one) to the salt solution.

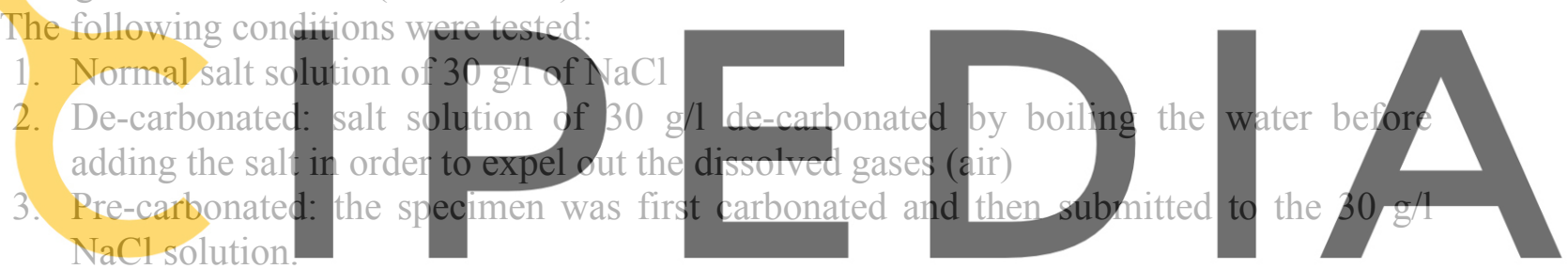

When the specimens were cut to have the duplicate samples, two of the set (1 and 2) were

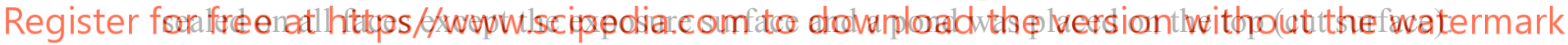
in set-1 non-de-carbonated and in set 2 the solution was previously de-carbonated.

The other two duplicates of the set-3 were introduced in a carbonation chamber at $100 \%$ $\mathrm{CO}_{2}$ during 30 days. Then, the pond was placed on the cut surface with a normal solution of $\mathrm{NaCl}$ for 90 days. The pond was covered with a plastic film in order to minimize the progressive uptake of air by the solution during the exposure time.

Finally, when removing the pond, chloride profiles were made by grinding the specimens mm-per-mm and analysing the chlorides (total and water soluble) (Castellote et al., 2002; Castellote et al., 2002a; Cen 12390-11 2009). In the powder samples it was also determined the portlandite and carbonate contents by Differential Thermal Analisys (DTA).

\section{Results}

The chloride profiles of the three set of specimens are given in Figure 1. The three profiles differ significantly, mainly when the sample was previously carbonated. Each profile is the average of the duplicate samples, which gave very similar ones. The symbols here and in the rest of Figures are: Set-1 $(\mathrm{NaCl})$, set-2 $\left(\mathrm{Cl}+\mathrm{CO}_{2}\right)$, set-3 $\left(\mathrm{CO}_{2}\right)$. 
All the profiles of the three set show a maximum some mm far from the concrete surface. Contrary to what was expected the highest chloride concentration is shown by the set- 2 with non-de-carbonated solution. The smallest chloride concentration is logically given by the sample previously carbonated, although the high difference between total and water-soluble chlorides indicates that there is significant amount of bound chlorides.

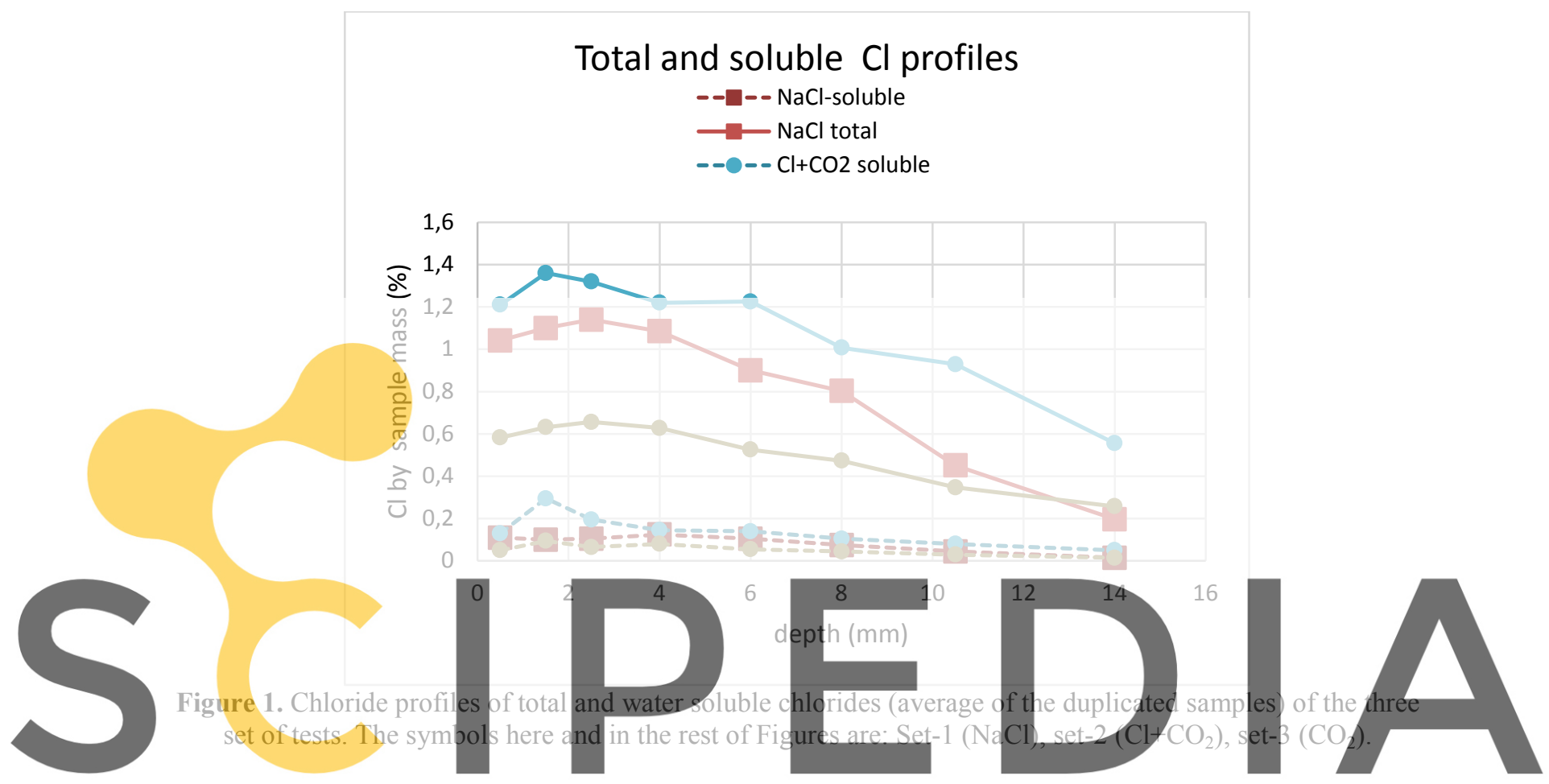

In Figure 2 is given the relation between the soluble chlorides (named free in the Figures)

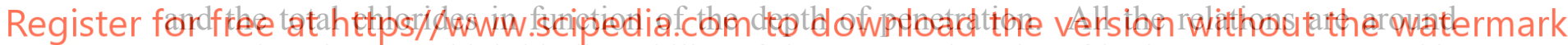
$0.1 \%$ denoting very high binding ability of the cement, in spite of its low $\mathrm{C}_{3} \mathrm{~A}$ content. This binding is attributed to the chloride adsorption in the C-S-H gel and in the portlandite (Florea and Brouwers, 2012). It is interesting to notice that the relation is not constant because it decreases with the depth and it is a bit smaller in the case of the carbonated specimen. Finally, it is important also to notice that the highest value of soluble chlorides appear in the case of normal salt solution (non-decarbonated set-2) in the depth where the maximum appears and that, therefore, it is the reason of the higher total value. 


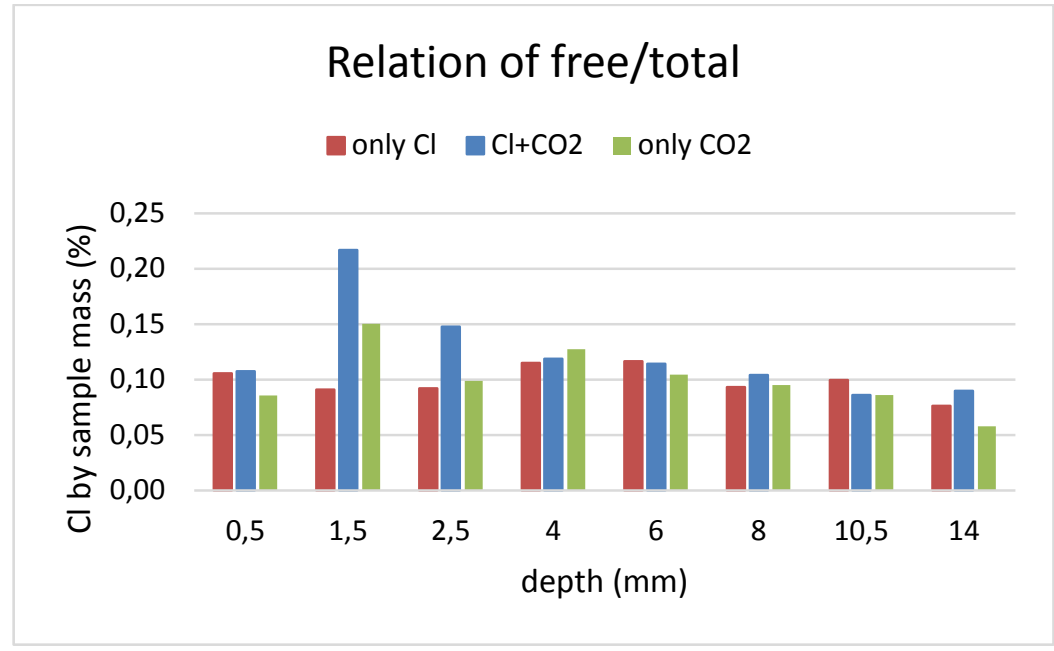

Figure 2. Ratios of free/ bound chlorides in the three set of specimens.

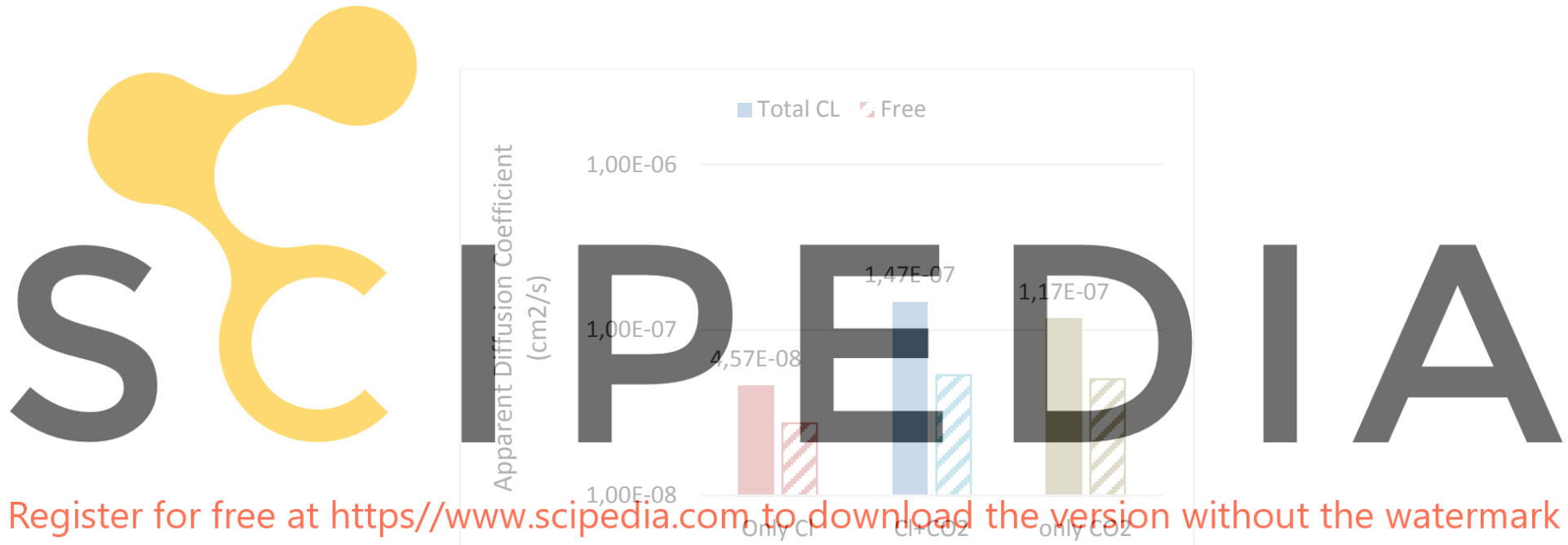

Figure 3. Apparent Diffusion Coefficient calculated through the fitting of the error function equation into the profiles of Figure 1.

The diffusion coefficient and the surface concentration deduced from the fitting of the error function equation into the profiles, are given in Figures 3 and 4 respectively. The diffusion coefficients, $\mathrm{D}_{\mathrm{ap}}$, differ between the three set and also between fitting into the soluble and total chloride profiles, being smaller if fitted into the soluble chloride profile. The highest value of both parameters is given by the normal salt solution (set-2) and the smallest surface concentration but highest $\mathrm{D}_{\mathrm{ap}}$, in the case of the pre-carbonated specimen (set-3). 


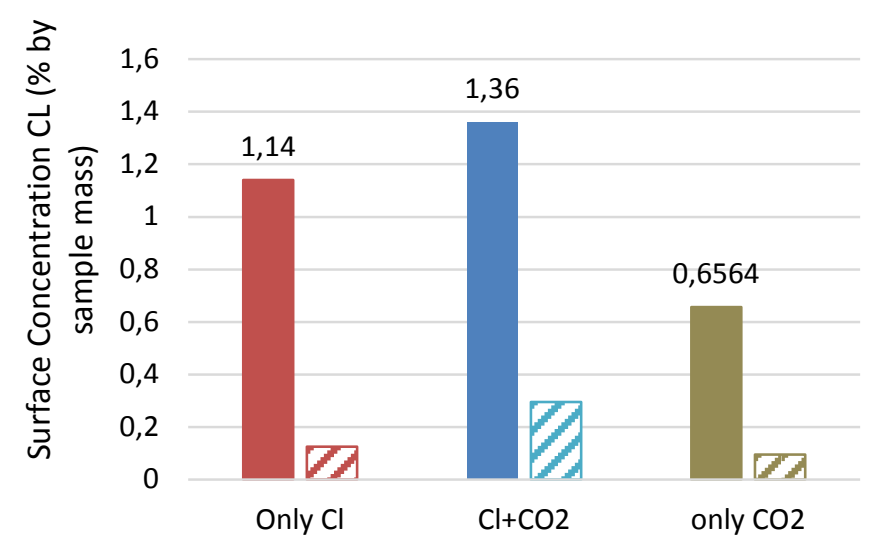

Figure 4. Chloride surface concentration calculated through the fitting of the error function equation into the profiles of Figure 1

The results of the Portlandite and Calcite contents calculated from DTA analysis are shown in Figure 5 to 7 together with the chloride profiles. One first surprise is that the content of calcite in the surface of the concrete is a bit higher in the sample with de-carbonated solution, which indicates that the carbon dioxide has been uptake during the exposure of the 90 days in spite of the covering of the pond. Other observation is that the calcite profile is decreasing

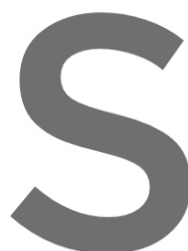
towards the interior an dioxide is reacting at carbonated specimen decreasing towards the carbonated and it shows th
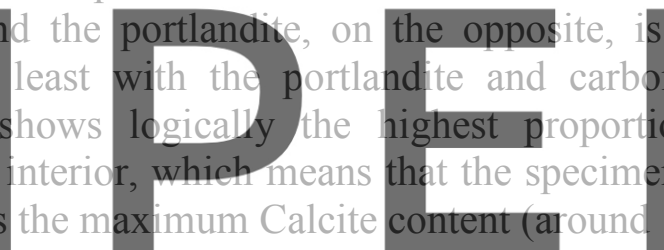

The presence of calcite seems to have a direct relation to the production of the maximum in Register for free at https//www.scipedia.com to download the version without the watermark

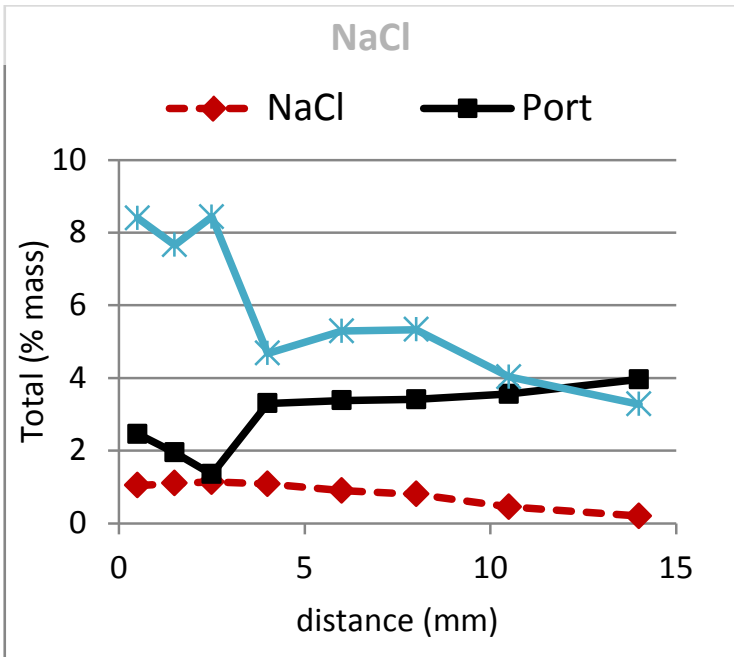

Figure 5. Profiles of calcite, portlandite and total chlorides in set-1.

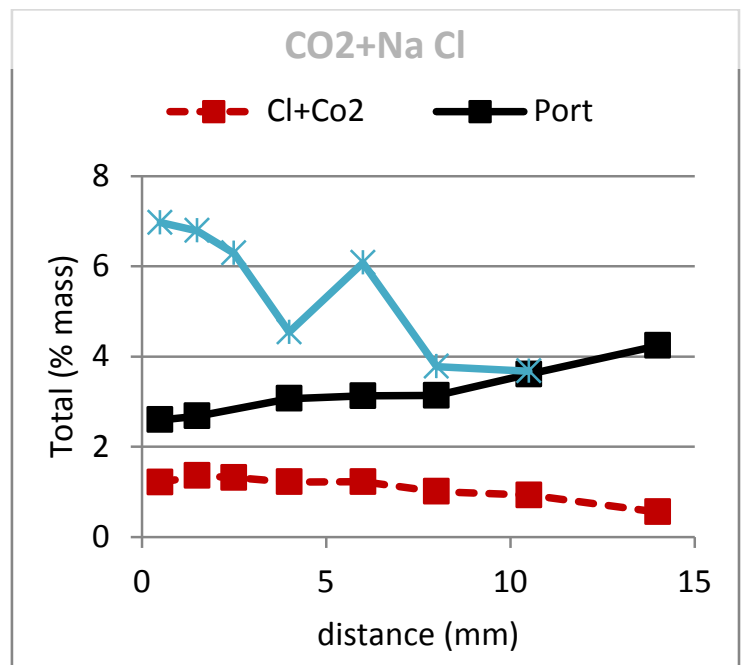

Figure 6. Profiles of calcite, portlandite and total chlorides in set-2. 


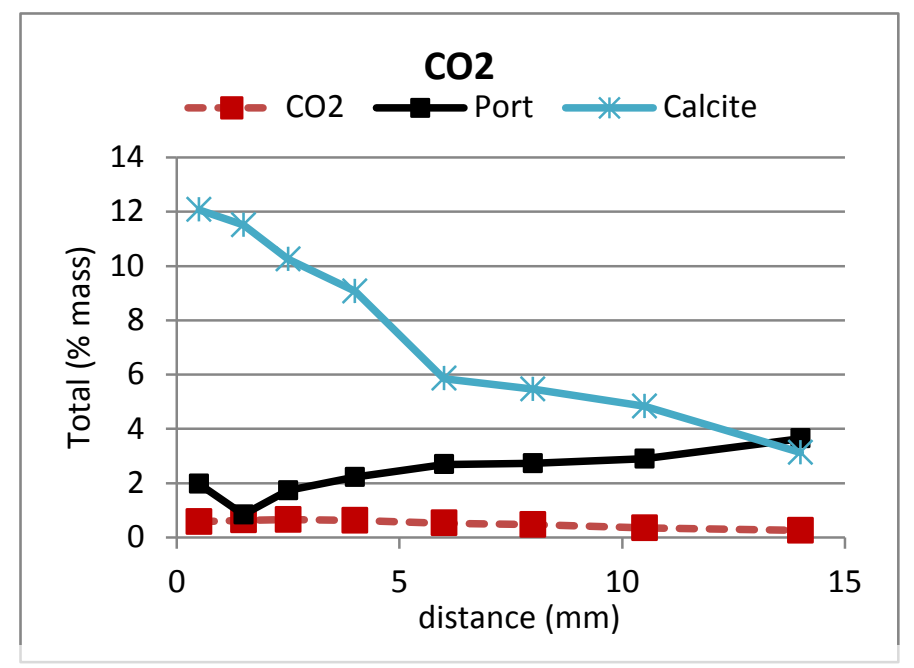

Figure 7. Profiles of calcite, portlandite and total chlorides in set-3.

\section{Discussion}

The results enable to emphasize that carbonation is not only produced in unsaturated conditions, but it is also produced by the air dissolved in the water in contact to a saturated concrete.

With respect to the 1 commented further the 2). In set-2, the salt sol The bicarbonate will be transformed into carb on high alkalinity of the p the calcium bicarbonate is soluble and does not precipitate carbonate/bicarbonate at $\mathrm{pH}=10.4$. The bicarbonates can diffuse inside the sample producing

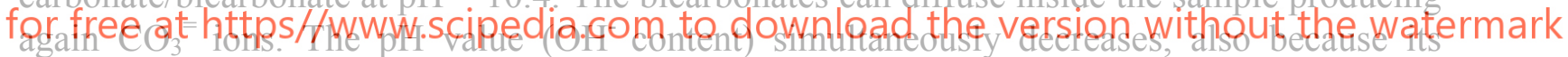

\section{leaching out due to the external pH value is neutral.}

In set-2 with the standard sait solution, the chiorides and the bicarbonate entry simultaneously but the chlorides have higher ionic mobility. They penetrate first and bind with the hydrated phases (Afm phases, C-S-H gel and portlandite). The bicarbonate arriving later, on one hand, exchanges with the chloride in the hydrated phases releasing free chlorides to the pore solution, which move inwards again. They cannot move backwards due to the concentration gradient. On the other hand, the bicarbonates become carbonate ion due to the high alkalinity, precipitating $\mathrm{CaCO}_{3}$. The $\mathrm{CaCO}_{3}$ nucleation will physically cover the pore walls, decreasing the reactive sites for the chloride in the hydrated phases. Consequently. The total chloride decreases in the carbonated zone.

The maximum is produced because the pushing inside of the released $\mathrm{Cl}$ by the carbonation from the bound ones. The $\mathrm{CO}_{2}$ in the salt solution acts as an intensifier of the chloride penetration. The profile advances due to the gradient between the maximum and its farther front inside the concrete. All the profile moves a step forward. New chlorides have to penetrate from outside to maintain the equilibrium with the bound chlorides to fulfil the so called chloride isotherm (Tang and Nilsson, 1993), all along the profile. That is, the 
progressive carbonation of the skin decreases its bound and total chloride content, but does not stop the chloride penetration due to the need to maintain the Bound/ free chloride equilibrium, although this carbonation perhaps reduces with the time the amount of penetrating chlorides and it is one of the reasons of the "aging" effect (decrease with time of the diffusion coefficient). The external carbonated zone acts as a "filter" by the progressive decreasing the chloride flow.

In set-1 with the de-carbonated salt solution, the profile shape is similar than in set-2, but the total amount of chlorides is smaller. If the bicarbonates are in smaller amount, the carbonation will be less intense and the amount of exchanged chlorides in the interior of the pores will be smaller, and so the released free chlorides. The maximum in the profile is produced, but the total chlorides are smaller.

In the case of the pre-carbonated specimen (set-3), the profile shape is different than the other two set, because it is flatter. The total chloride is even smaller than in set-2 due to the reactive sites, from the beginning, are in much smaller amount. Also less chloride amount penetrate perhaps due to the smaller porosity produced by the carbonation. In addition, the pH of the pore solution is neutral, and then the bicarbonate will not be converted into carbonate when entering in the pore network. The neo-formed calcite will be in less amount. The specimens have more calcite due to the previous carbonation.

In this set-3, the pre-carbonation has not being complete enough to stop binding and then, it is remaining Afm, C-S-H and portlandite that are able to adsorb chlorides (Figure 1). This behaviour helps to conclude that the carbonation influences in a different manner the chloride penetration when it penetration. One main carbonation previous y decreases the $\mathrm{pH}$ of that of the saltsolution, in the case of the $\mathrm{CO}$ solution is initially allaline.
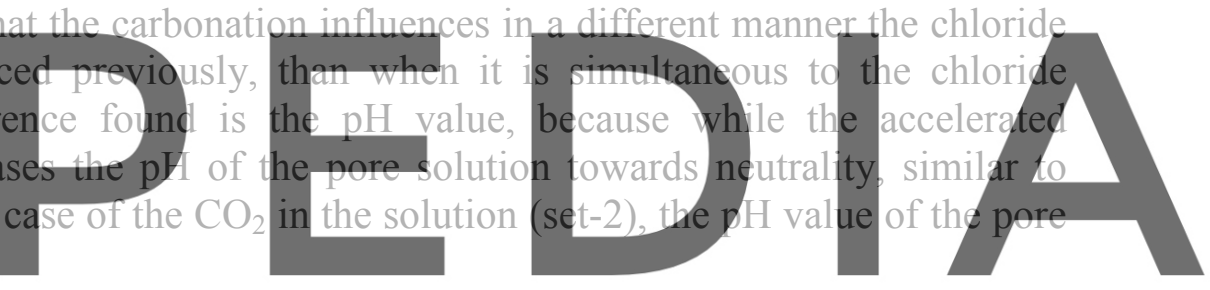

In summary, not only the carbonation itself, but the equilibrium carbonate/bicarbonate,

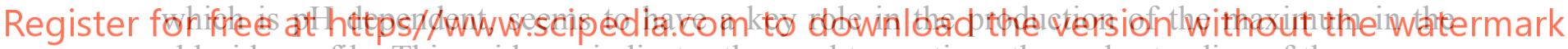
chloride profile. This evidence indicates the need to continue the understanding of the process by studying the diffusion of the bicarbonate ion into the pore network, because it seems that the $\mathrm{HCO}_{3}{ }^{-}$as being soluble, is a competitor of the chloride to be bound in the reactive sites and because it is evolving to carbonate at high $\mathrm{pH}$ level, inducing the precipitation of calcium carbonate, physically affecting the contact area of the pore solution and the reactive hydrated phases.

\section{Conclusions}

- The air $\left(\mathrm{CO}_{2}\right)$ content of the salt solution influences the chloride profile and in consequence, the calculated diffusion coefficient.

- The most aggressive condition (enabling higher chloride penetration) opposite to what was the hypothesis, is that of the standard salt solution (non-de-carbonated). This enables to confirm that present testing conditions for natural diffusion, are the most convenient ones.

- The pre-carbonation of the specimen leads into smaller amount of penetrated chlorides and a flatter profile. 
- The production of the maximum in the chloride profile is attributed to the higher ionic mobility of the chlorides with respect to the carbonate/bicarbonates. Chloride binding is produced before the advance of the $\mathrm{CO}_{3}{ }^{=} / \mathrm{HCO}_{3}{ }^{=}$. When carbonation is produced behind the chloride penetration, chlorides are released that move forward making the profile to advance.

- In the external layers of concrete the bound chlorides are smaller due to the carbonation. This mechanism may contribute to the known as "aging effect".

- Bound chlorides have to be in equilibrium with the free chlorides given by the corresponding isotherm.

- Previous carbonation decreases the amount of reactive sites for the chlorides although may not fully exhaust them.

- A maximum is also noticed in the pre-carbonated specimen attributed to the same mechanism than the non-carbonated as in the material remain sites for chloride adsorption.

Acknowledgements

The author is grateful to Prof. A. Moragues of the Faculty of Civil Engineering of the Polytechnic University of

Madrid for the fruitful discussions on the subject and the analysis made on the samples.

References

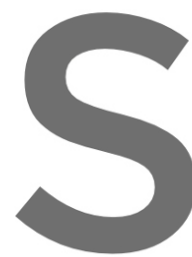

Andrade, C. Díez, J, and Diffusion in chloride con

Andrade, C. Climent, M. and surface concentratio Structures, 48, 863-869.

Baroghel-Bouny, V., Wang
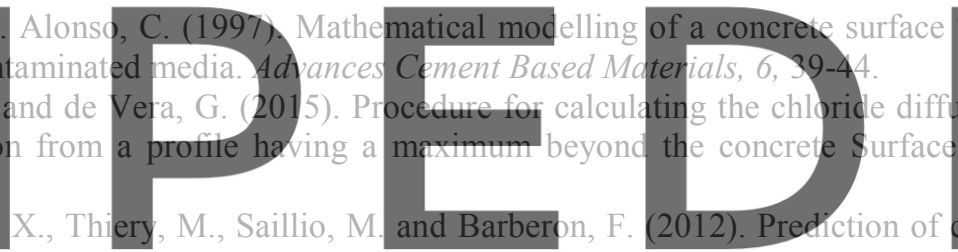

isotherms of cementitious materials by analytical model or numerical inverse analysis. Cement and Concrete Research, 42, 1207-1224

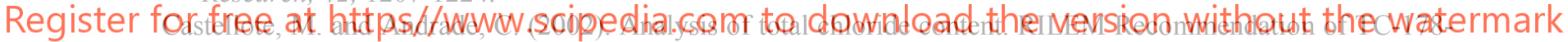
Testing and modelling chloride penetration in concrete. Materials and Structures, 35, 583-585.

Castellote, M. and Andrade, C. (2002a). Analysis of water soluble chloride content in concrete. RILEM Recommendation of TC-178. Testing and modelling chloride penetration in concrete. Materials and Structures, 35, 586-588.

CEN/TS 12390-11: 2009. Testing hardened concrete. Determination of the chloride resistance of concrete. Unidirectional diffusion.

Florea, M.V.A. and Brouwers, H.J.H. (2012). Chloride binding related to hydration products Part I: Ordinary Portland Cement and Concrete Research, 42, 282-290.

Galan, I. and Glasser, F.P. (2015). Chloride in Cement. Advances in Cement Research, 27(2), 63-97.

Lianfang, L. and Sagües A.A. (1999). Effect of chloride concentration on the pitting and repassivation potentials of reinforcing steel in alkaline solutions. NACE Corrosion Paper, 567.

Ngala, V.T. and Page, C.L (1997). Effects of carbonation on pore structure and diffusional properties of hydrated cement pastes. Cement and Concrete Research, 27(7), 95-1007.

Qiang, Y., Caijun, S., De Schutter, G., Dehua, D. and Fuqiang, H. (2011). Chloride Ion Concentration on the Surface of Cement-Based Materials in Chloride Solutions. Journal of the Chinese Ceramic Society, 39(3), 544-549.

Tang, L.P. and Nilsson, L.O. (1993). Chloride binding capacity and binding isotherms of OPC pastes and mortars. Cement and Concrete Research, 23, 247-253. 